\title{
Validating the Ffowcs Williams and Hawkings acoustic analogy implementation in Antares
}

\author{
Danilo Di Stefano, Aldo Rona $†$ Edward Hall; Christopher L. Morfey§ \\ Department of Engineering, University of Leicester, Leicester, LE1 7RH, England \\ Guillaume Puigt \\ CERFACS, 42, av. Coriolis, 3105\%, Toulouse, Cedex, France
}

\begin{abstract}
Progress is presented in the development of a Ffowcs Williams and Hawkings (FW-H) acoustic analogy post-processor for time-resolved Computational Fluid Dynamics (CFD) simulations. The tool is coded in Python and embedded in Antares, a post-treatment package (developed by Cerfacs, France) of wide access and usability for the scientific community. Tests on monopoles, dipoles, and quadrupoles show good predictions of pressure fluctuation and directivity against reference analytical results, validating the software for applications to stationary elementary sources. The proximity of these sources to a corner of the FW-H integration surface is shown to adversely affect the predictions and good agreement with the benchmark sound field is restored by an appropriate sizing and placement of the FW-H surface. Guidelines for obtaining predictions that are substantially independent of the size and placement of the FW-H surface are provided in terms of the spatial resolution of the discretised FW-H surface.
\end{abstract}

\section{Introduction}

Current advances in Computational Fluid Dynamics (CFD) and Computational Aero-Acoustics (CAA) $\checkmark$ are facilitated by the sharing of benchmark computations and experimental data across the community on a royalty-free non-commercial base. These initiatives are strengthened by the increasing availability of open-source software that accelerates the availability and the use of advanced numerical methods across the research community. On these premises, the authors aim to develop a noise extractor code that can be used and improved by a wide number of researchers in the aero-acoustic field. The code is structured for good portability across different computational platforms in order to be able to couple the tool to a wide range of CFD solvers. Bespoke data structure interfaces are required between the CFD software and the Ffowcs Williams and Hawkings (FW-H) code and effort has been put towards minimising the programming cost of this interface.

A FW-H acoustic analogy formulation, implemented in Python, enables the software to predict the acoustic pressure fluctuation on observers placed at an arbitrary distance from the acoustically active part of the flow, from the CFD flow prediction history and the FW-H surface topology. In this way the CFD and CAA solvers can be written independently from one another, which enables greater flexibility in the design of coupled CFD-CAA tools.

At each time step, the FW-H post-processor receives as input the unsteady hydrodynamic flow field in terms of density, static pressure and flow velocity. An integration is then performed on a FW-H surface (figure 1(a)) that is defined a-priori by the user and is test-case specific. Next, the software builds a segment of the time-history of the radiated noise to specific observers, by estimating at each time step the contribution

\footnotetext{
* Marie Curie Fellow.

${ }^{\dagger}$ Thermofluids Senior Lecturer, MAIAA.

${ }^{\ddagger}$ ITN Technical Manager.

$\S$ Visiting Professor.

ISenior researcher
} 
to the acoustic pressure fluctuation. The different contributions are finally summed up by synchronising their advanced time ${ }^{1}$ to obtain the acoustic pressure history.

The formulation of the FW-H acoustic analogy described by Casalino ${ }^{1}$ is used to obtain the results of Sections A-D. The analogy with an acoustic medium at rest described by Lighthill ${ }^{2}$ is revisited by Casalino ${ }^{1}$ and an integral solution of the FW-H equation ${ }^{3}$ in the advanced time formulation ${ }^{4}$ is obtained. ${ }^{1}$ The $\mathrm{FW}-\mathrm{H}$ equation is a generalization of the Lighthill acoustic analogy that takes into account the presence of bodies in arbitrary motion. ${ }^{3}$

As sketched in figure 1(a), in this generalization a closed surface (FW-H integration surface) determines three different regions in which the fluid is assumed to have different properties. ${ }^{3}$ Brentner and Farassat discussed the integration surface requirements and propose a permeable/porous formulation in which the closed surface does not coincide with a physical body ${ }^{5,6}$

The flow outside this surface is modelled by the Navier-Stokes equations. Inside the closed surface, the conservation laws are assumed not to apply and the flow state is specified arbitrarily. In order to maintain the discontinuity generated in such a way across the surface, mass and momentum sources are distributed over the closed integration surface. ${ }^{3}$ A distribution of quadrupole-like sources in the volume outside the integration surface completes the noise radiation model by taking into account of non-linear effects. ${ }^{2,3}$ This process is carried out from the time-resolved estimates of pressure, density and velocity on the FW-H integration surface, obtained independently, for instance, by CFD.

The equations of conservation of mass and momentum are taken as valid everywhere in an exterior flow domain free from solid boundaries. The theory of generalized functions is used to combine these equations in the form of an inhomogeneous wave equation. Therefore the FW-H surface needs to enclose all the solid bodies interacting with the flow. This embedding procedure is detailed by Farassat ${ }^{7}$ and only a brief outline is given herein for conciseness.

Let $g(\mathbf{x}, t)=0$ be the equation describing the moving control surface of figure 1(a), whose points move at velocity $\mathbf{v}(\mathbf{x}, t) .{ }^{1} g$ is defined to satisfy the property $\nabla g=\hat{\mathbf{n}}$ on the surface, where $\hat{\mathbf{n}}$ is the outward pointing unit normal vector. Following this procedure, the flow is partitioned into three regions according to the value of $g$, as shown in figure 1(a). A point enclosed by the integration surface satisfies $g<0$ and this flow field portion can be replaced by a quiescent fluid. A point outside the integration surface satisfies $g>0$ and lies in an acoustically perturbed fluid. The fluid motion is therefore discontinuous across $g=0$. Mass and momentum sources are hence distributed on the surface $g(\mathbf{x}, t)=0$, which allows the conservation laws across $g(\mathbf{x}, t)=0$ to be satisfied.

In implementing this formulation, an object oriented language is used (Python 2.7.9) and the tool is embedded in Antares 1.4.0. ${ }^{8}$ This represents an innovative approach in flow simulations, where a high-level programming language, such as Fortran, is usually preferred. The Antares software package presents many advantages to support the development of the FW-H post-processor. The package contains numerical tools which enable steady and unsteady flow analyses in real time and/or a posteriori and flow visualization. Antares also supports popular structured and unstructured CFD geometries and solutions, which provides a significant usability advantage in the aero-acoustic field. Besides this, Antares is an unrestricted, royalty-free software, freely obtainable subject to a license agreement for copyright purposes.

The new FW-H post-processor is expected to be further developed and used in collaboration with Cerfacs, France, which coordinates the development of Antares. This aims to keep the software up-to-date and continuously improved, including, for instance, new features and adapting the implementation to the new requirements of researchers in the field, so that the benefit of the overall aero-acoustic community can be pursued.

A reliable validation process of the software is necessary and this has been undertaken by the authors. The first stage of this process, with sample results, was presented by Di Stefano et al. ${ }^{9}$ The predictive ability of the code was shown by applying the FW-H acoustic analogy implementation to three test cases: a monopole source, a dipole source and a subsonic jet flow modelled by Bogey and Bailly. ${ }^{10}$ Pressure fluctuations predicted by the tool were compared against reference solutions for each of the three test cases. These comparisons showed a satisfactory quantitative match in each test and built up confidence in the reliability of the code for estimating near-field and far-field noise radiation from both simple sources and more complex flows.

This paper presents the second stage of the validation process, aimed at demonstrating the ability of the code to model the radiation characteristics of compact sources located off-centre inside the FW-H integration surface that can have a more directive, multi-lobar radiation pattern than the previous test cases. Three 


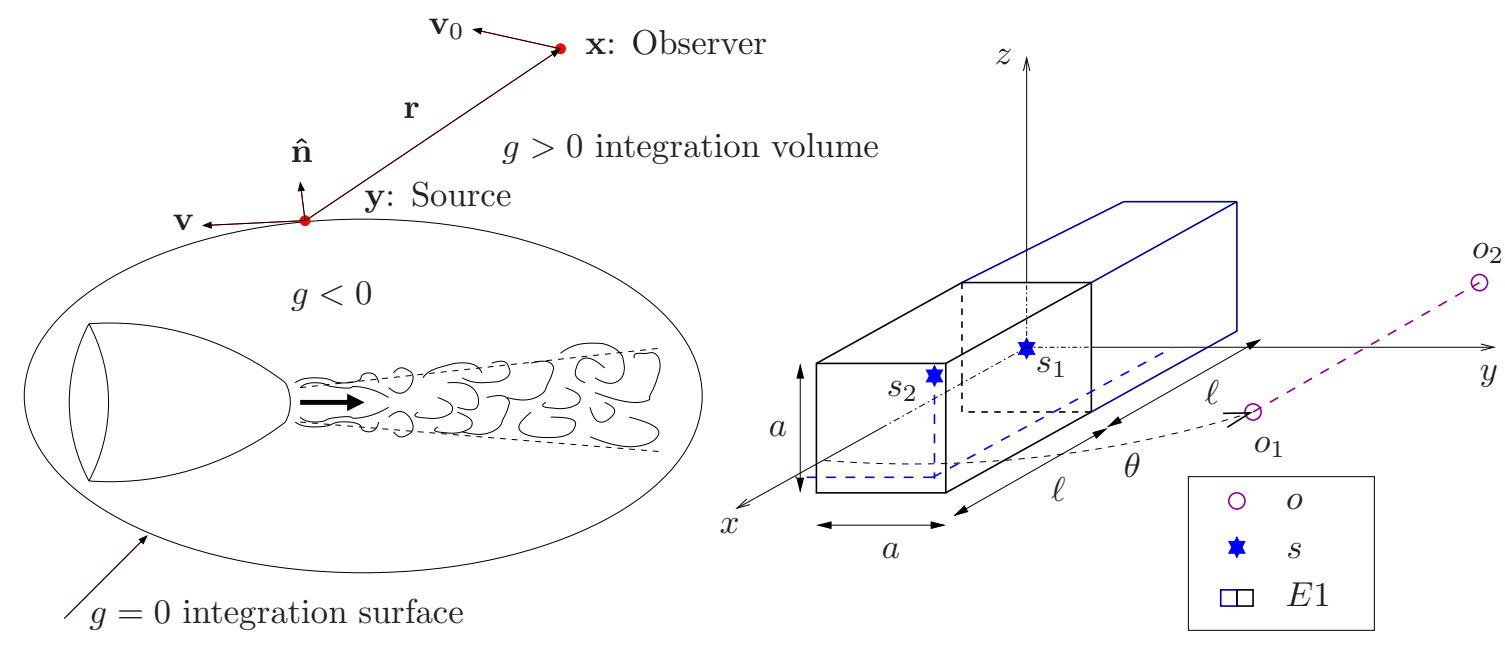

(a) Radiation in an unbounded flow.

(b) Prismatic integration surface.

Figure 1. Schematic of Ffowcs Williams and Hawkings surfaces.

kinds of elementary sources are considered, monopoles, dipoles and quadrupoles, which are general source models for decomposing the radiation of unsteady flows with and without the presence of solid boundaries.

The prediction of the directivity for such point sources is tested in Sections A and B and compared to reference analytical solutions. ${ }^{11}$ To estimate the directivity pattern, a circular array of far-field observers on the $z=0$ plane is considered. In figure 1(b), two observers from this circular array are shown. The array is centred at the origin of the Cartesian reference system and has a radius $r$ equal to 10 times the acoustic wavelength $\lambda$.

All tests use FW-H integration surfaces of the same topology, as sketched in figure 1(b). A square prism of cross-section $a \times a$, centred at the origin, encloses the noise sources. The length normal to the crosssection $a \times a$ of the FW-H prism extends symmetrically about the origin of the Cartesian axes over the range $-\ell \leq x \leq \ell$, as per configuration E1 in figure 1(b). Two source positions are defined, respectively $s_{1}$ and $s_{2}$. The source $s_{1}$ is placed at the origin in order to reproduce the reference directivity pattern ${ }^{11}$ characterizing its radiation. The location of source $s_{2}$ is chosen to test the robustness of the CAA scheme to $s_{2}$ being proximal to the corner of the prismatic FW-H surface, which represents a potential source of numerical error in the application of acoustic analogy approaches.

For each source position $s_{1}$ and $s_{2}$, pressure time-histories are compared against the reference solution at two observer positions, $o_{1}$ and $o_{2}$ shown in figure $1(\mathrm{~b})$, placed symmetrically about the $y-z$ plane. The coordinates of the observers are respectively $\mathbf{x}_{1}=(8.92,4.53,0.00) \lambda$ and $\mathbf{x}_{2}=(-8.92,4.53,0.00) \lambda$, corresponding, respectively, to an azimuthal angle of $\theta=27^{\circ}$ and $\theta=180^{\circ}-27^{\circ}$ from the $x$-axis.

In section $\mathrm{C}$, the effect of a change in the position of the $\mathrm{FW}-\mathrm{H}$ integration surface is investigated. Specifically, the size of the FW-H surface is changed in order to analyse the effect of an increased distance between the source and the surface on the acoustic results. This test aims to show the independence of the solution on the integration surface position and size, as it would be expected in a FW-H acoustic analogy approach that uses the porous surface formulation (Brentner and Farassat ${ }^{5}$ ).

Section D reports a parametric analysis of the effect of the presence of sharp corners in the FW-H integration surface. The error between the numerical prediction and the analytical solution is investigated by moving the source along the diagonal of the FW-H prism and by changing the numerical spatial discretization.

Details of the mathematical formulation adopted and of its numerical implementation are reported in Di Stefano et al. ${ }^{9}$ and are not repeated herein for brevity. 


\section{Results}

\section{A. Omnidirectional sound source}

The first source considered is a monopole of sound power level $L_{W}=96.83 \mathrm{~dB}$ re $1 \mathrm{pW}$ which radiates at a frequency $f=5.67 \mathrm{kHz}$. This acoustic power gives a Sound Pressure Level (SPL) of $80 \mathrm{~dB}$ re $20 \mu \mathrm{Pa}$ at $33.33 \lambda$ from the source. The FW-H surface is used in configuration $E 1$ of figure 1 (b) with $a=2 \lambda$ and $\ell=\lambda$. A uniform spatial discretization is used with $N=80$ nodes in each spatial direction. The effect of the spatial discretization error of the FW-H surface on the numerical prediction was investigated by Di Stefano et al. ${ }^{9}$ and it was shown that $N=80$ provides estimates that are substantially mesh independent.

Figure 2(a) displays the predicted acoustic pressure fluctuation $p^{\prime}$ at the observer positions $o_{1}$ and $o_{2}$ for the off-centre source $s_{2}$ versus the benchmark analytical solution ${ }^{11}$ :

$$
p^{\prime}=B \sin (\omega t-k r)
$$

where $B=-\rho_{0} c_{0} q k(4 \pi r)^{-1}, \rho_{0}=1.225 \mathrm{~kg} / \mathrm{m}^{3}, c_{0}=340.25 \mathrm{~m} / \mathrm{s}, k=\omega / c_{0}, q=\left(8 \pi c_{0} W \rho_{0}^{-1} \omega^{-2}\right)^{1 / 2}$, $W=10^{L_{w}} p W$, and $\omega=2 \pi f$.

The analytical solution is represented by the black lines and labelled 'ref' in figure 2(a). A solid line denotes the analytical solution at the observer $o_{1}$, while a dash-dotted line represents the analytical solution at the observer $O_{2}$. Blue and red symbols are used respectively for the numerical predictions at observers $o_{1}$ and $o_{2}$.

The source $s_{2}$ is placed at $\mathbf{y}_{2}=(0.92,0.92,0.92) \lambda$, while the corner of the FW-H prism is at $(1.00,1.00,1.00) \lambda$. In figure $2(\mathrm{a})$, the prediction substantially matches the analytical solution in amplitude, phase and frequency at both observers $o_{1}$ and $o_{2}$ despite the small distance $d / \lambda=0.14$ between the source and the corner $(a / 2, a / 2, \ell)$ of the FW-H integration surface. The acoustic pressure fluctuation amplitude at observer $o_{1}$ is greater than that at $o_{2}$, due to the shorter source-observer distance.

Figure 2(b) shows the directivity pattern on a circular array centred at the origin of the reference system at $10 \lambda$ constant radial distance both for $s_{1}$ and $s_{2}$. The black lines represent again the analytical solution, while the symbols represent the numerical prediction. A substantial match is found between the analytical and the numerical predictions sound pressure directivity for both source positions. In figure 2(b), a symmetric pattern is obtained with the source placed at $s_{1}$. When the monopole is moved towards the corner of the prism, the symmetry is lost and an oval-shaped pattern is generated, with maximum amplitude at $\theta=45^{\circ}$, in the direction of the shortest distance from the source to the observer.

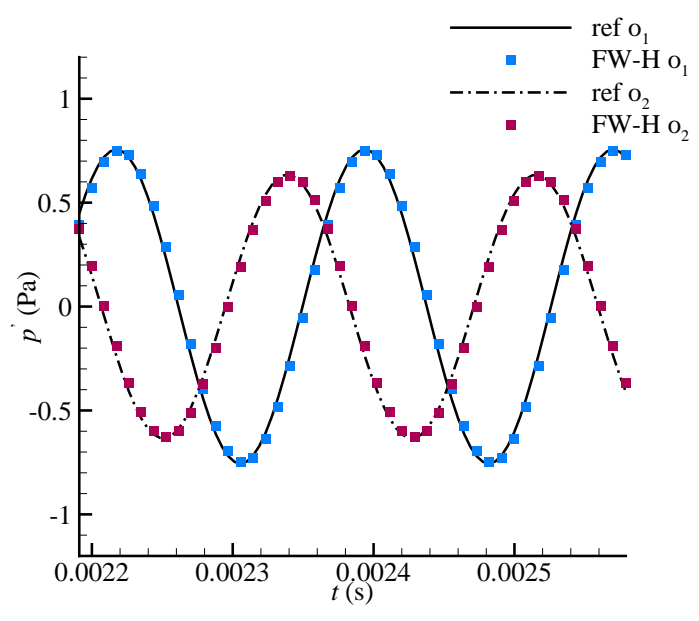

(a) Sound pressure fluctuation at $o_{1}$ and $o_{2}$ from the monopole located at $s_{2}$.

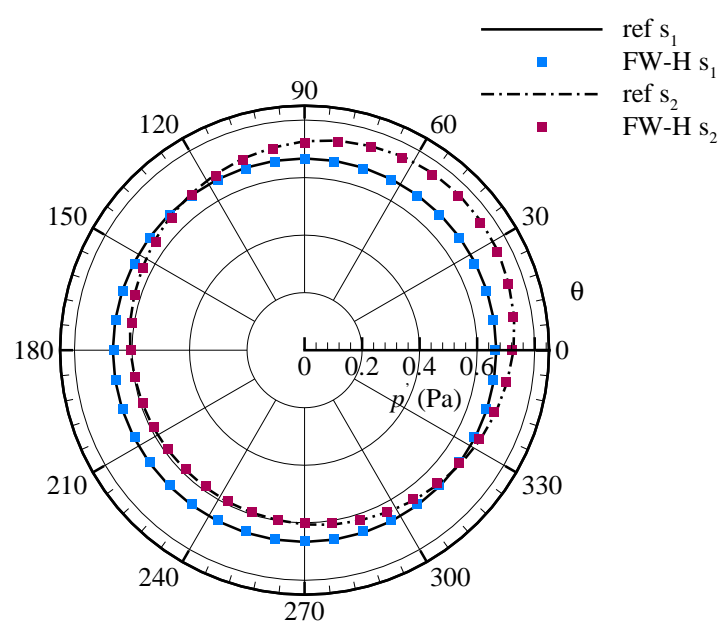

(b) Directivity pattern at $10 \lambda$ from the origin, from the monopole located at $s_{1}$ and at $s_{2}$.

Figure 2. Sound pressure fluctuations and directivity patterns from a $96.83 \mathrm{~dB}$ re $1 \mathrm{pW}$ monopole radiating at $f=5.67 \mathrm{kHz} . \lambda=0.06 \mathbf{m}, \ell=\lambda, a=2 \lambda$. 


\section{B. Directional sound sources}

Three directional sound sources are considered, these being a dipole, a longitudinal quadrupole, and a lateral quadrupole. The same discretisation of the $\mathrm{FW}-\mathrm{H}$ surface and the same observer positions are used as in section A. The results are shown in figures 3-5(a) where the same notation of figure 2 is adopted.

Firstly, a $y$-axis dipole sound source is tested defining its strength so that the same $80 \mathrm{~dB}$ re $20 \mu \mathrm{Pa}$ is obtained on the $y$-axis, 33.33 $\lambda$ away from the source, as in section A. The acoustic pressure fluctuation predicted at $o_{1}$ and at $o_{2}$ radiating from $s_{2}$ is shown in figure 3(a) together with the reference analytical solution

$$
p^{\prime}(\mathbf{r}, t)=B \frac{k(\mathbf{r} \cdot \boldsymbol{\epsilon})}{r}\left[\cos (\omega t-k r)+\frac{\sin (\omega t-k r)}{k r}\right],
$$

where $\epsilon$ is the dipole direction vector. ${ }^{11}$ A good engineering match is shown between the prediction and the reference solution. Away from the $y$-axis, the dipole radiation exhibits a lower amplitude at the same distance from the source compared to the monopole, which is the sound source with the highest strength for the same sound power level.
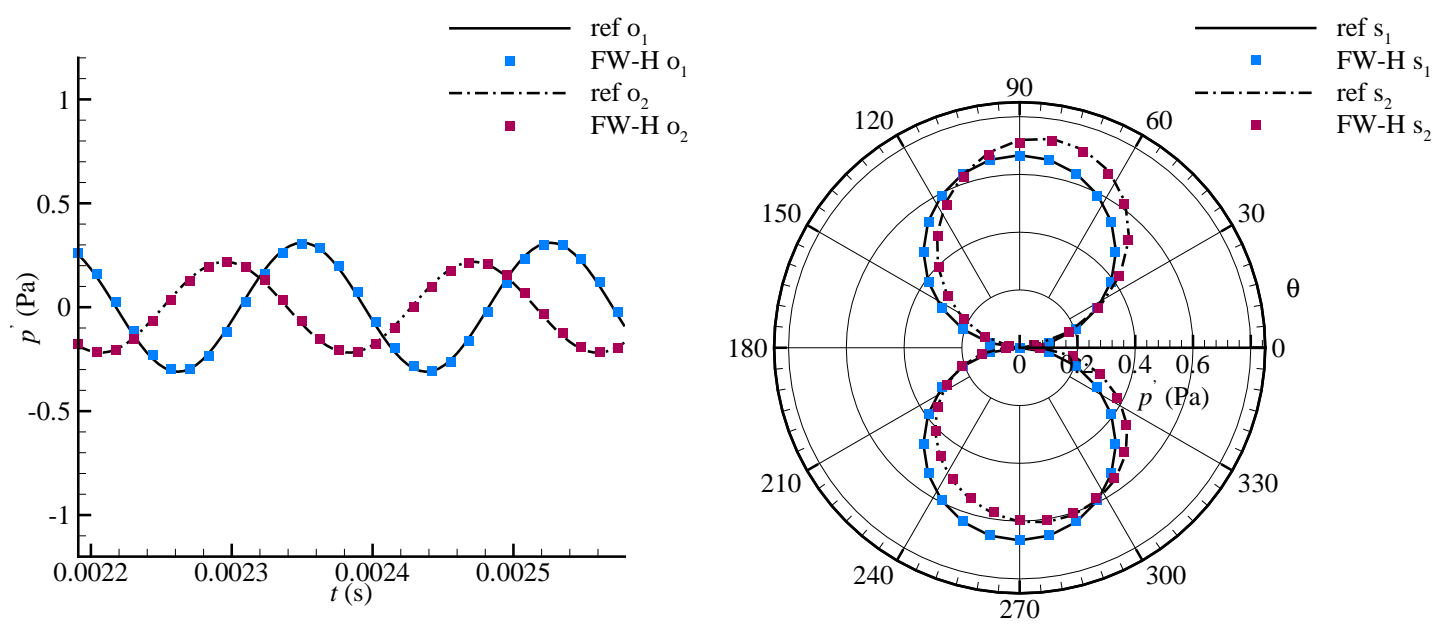

(a) Sound pressure fluctuation at $o_{1}$ and $o_{2}$ from the (b) Directivity pattern at $10 \lambda$ from the origin, from the dipole located at $s_{2}$.

dipole located at $s_{1}$ and at $s_{2}$

Figure 3. Sound pressure fluctuations and directivity patterns from a $96.83 \mathrm{~dB}$ re $1 \mathrm{pW}$ dipole radiating at $f=5.67 \mathrm{kHz} . \lambda=0.06 \mathbf{m}, \ell=\lambda, a=2 \lambda$.

In the directivity pattern reported in figure 3(b), the characteristic figure-of-eight shape of the dipole radiation is correctly reproduced by the code for both $s_{1}$ and $s_{2} . s_{1}$ gives a symmetric radiation about the origin. The location of $s_{2}$ breaks this central symmetry, giving a stronger radiation in the $\theta=45^{\circ}$ direction, which is the direction of minimum distance between source and observer.

The second directional sound source test case is a $y$-axis longitudinal quadrupole that can be approximated by two dipole sources with equal strength and in-line axes. The analytical solution for the far-field pressure perturbation ${ }^{11}$ is:

$$
\begin{aligned}
p^{\prime}(\mathbf{r}, t) & =-B_{1} \frac{(\mathbf{r} \cdot \boldsymbol{\epsilon})^{2}}{r^{2}}\left[\frac{3}{k r} \cos (\omega t-k r)+\left(\frac{3}{(k r)^{2}}-1\right) \sin (\omega t-k r)\right] \\
& +B_{1} \epsilon^{2}\left[\frac{1}{k r} \cos (\omega t-k r)+\frac{1}{(k r)^{2}} \sin (\omega t-k r)\right]
\end{aligned}
$$

where $B_{1}=\rho_{0} c_{0} q k^{3}(4 \pi r)^{-1}, \boldsymbol{\epsilon}$ is the direction of the longitudinal quadrupole ${ }^{11}$ and $\epsilon^{2}=|\boldsymbol{\epsilon}|^{2}$.

Figure 4(a) shows the pressure fluctuation relative to observers $o_{1}$ and $o_{2}$ obtained using a different FW-H surface size than the size used for predicting the radiation from monopole and dipole sources. As 

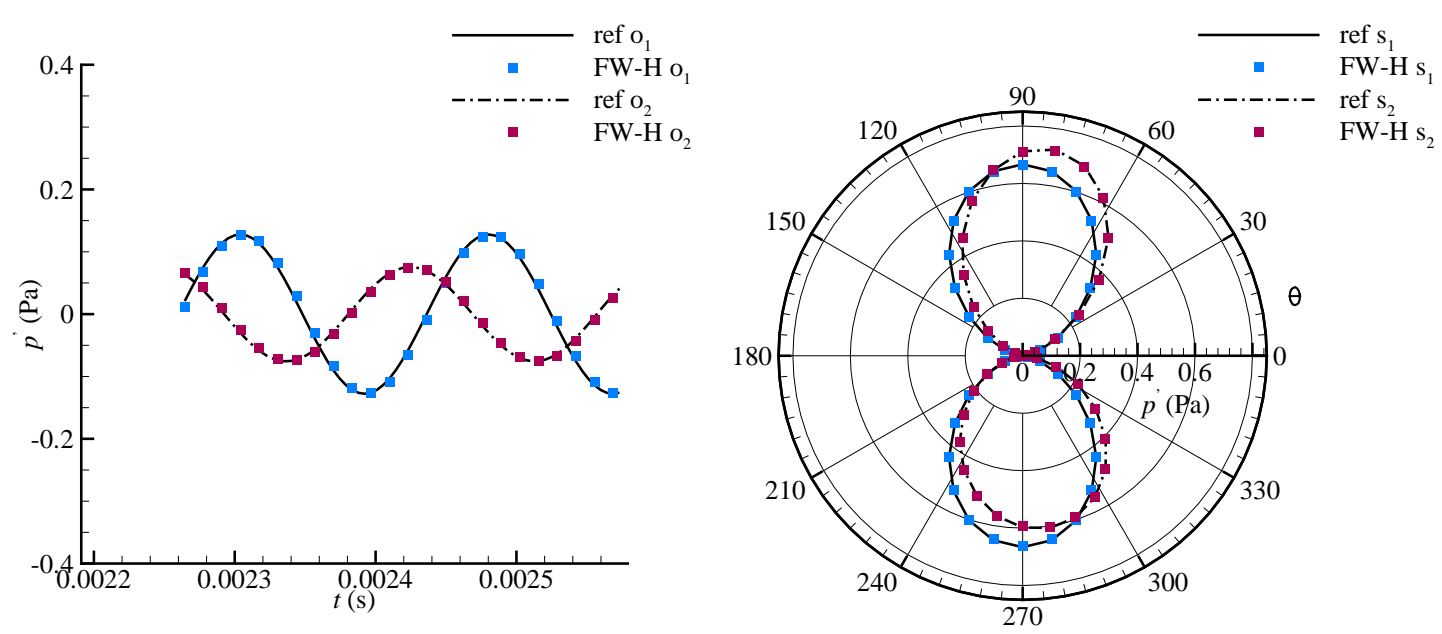

(a) Sound pressure fluctuation at $o_{1}$ and $o_{2}$ from the lon- (b) Directivity pattern at $10 \lambda$ from the origin, from the gitudinal quadrupole located at $s_{2}$.

longitudinal quadrupole located at $s_{1}$ and at $s_{2}$.

Figure 4. Sound pressure fluctuations and directivity patterns from a $96.83 \mathrm{~dB}$ re 1pW longitudinal quadrupole radiating at $f=5.67 \mathrm{kHz} . \lambda=0.06 \mathrm{~m}, \ell=1.17 \lambda, a=2.34 \lambda$.

reported in section $\mathrm{C}$, for the quadrupole test case, the distance between the source and the corner of the FW-H surface of section A is too short to be able to correctly capture the noise radiation. A modification to the size of the surface is required and both the cross-section and the $x$-axis dimensions of the surface are increased respectively to $\ell=1.17 \lambda$ and $a=2.34 \lambda$. This change allows the recovery of a good match between the analytical and the numerical solutions, as shown by figure 4(a).

Figure 4(b) reports the directivity patterns relative to the quadrupole source obtained with the modified FW-H surface. The longitudinal quadrupole has a directivity pattern in a figure-of-eight shape in the farfield that is broadly similar to the one from a dipole. The prediction correctly reproduces this trend and a good match with the reference analytical solution is obtained. With the source $s_{1}$ placed at the origin, the radiation pattern is symmetric. With the source $s_{2}$ placed off the origin, the radiation pattern becomes asymmetric and the direction of maximum radiation is in the first quadrant $(\theta<\pi / 2)$.

The last test case is the numerical prediction of the radiating pressure field from a lateral quadrupole. The analytical expression of the pressure fluctuation ${ }^{11}$ is

$$
p^{\prime}(\mathbf{r}, t)=-B_{1} 4 \frac{\left(\mathbf{r} \cdot \boldsymbol{\epsilon}_{\mathbf{1}}\right)\left(\mathbf{r} \cdot \boldsymbol{\epsilon}_{\mathbf{2}}\right)}{r^{2}}\left[\frac{3}{k r} \cos (\omega t-k r)+\left(\frac{3}{(k r)^{2}}-1\right) \sin (\omega t-k r)\right],
$$

where $\boldsymbol{\epsilon}_{1}$ and $\boldsymbol{\epsilon}_{2}$ are the zero radiation directions for the lateral quadrupole. ${ }^{11}$

The numerical predictions from the FW-H scheme are compared in figure 5 (a) against the reference solution, Eq. (4). The quadrupole source $s_{1}$ placed at the origin generates a symmetric directivity pattern in the form of a clover leaf, which is a recognisable feature of the lateral quadrupole radiation pattern. ${ }^{11}$ By placing the sound source off-centre, as with $s_{2}$, the sound radiation pattern becomes asymmetric and the directivity lobe in the first quadrant increases, giving a peak radiation in the $\theta=\pi / 4$ direction. The opposite lobe in the third quadrant reduces in size and remains symmetric about the $\theta=5 \pi / 4$ line. The remaining two lobes become asymmetric and lean toward the first quadrant. The reduced source-observer distance in the first quadrant is responsible for the radiation peak in the $\theta=\pi / 4$ direction. By positioning the sound source on the $\theta=\pi / 4$ line, the quadrupole retains a symmetric radiation about this line. In spite of the greater complexity of the lateral quadrupole radiation pattern, figure 5(a) shows that the FW-H numerical prediction has correctly captured it, as the numerical predictions shown by the dots follows the analytical solutions shown by the lines. 


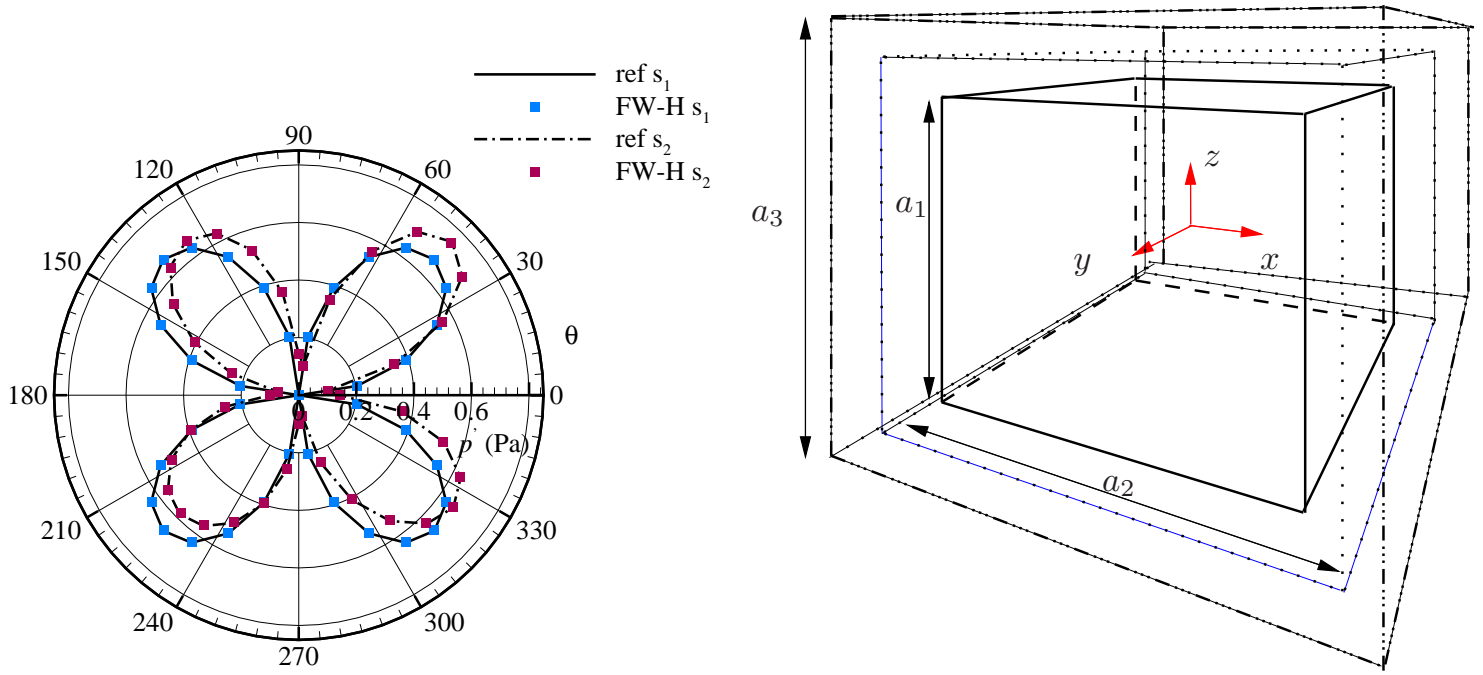

(a) Directivity patterns at $10 \lambda$ from the origin from a (b) Sketch of the three integration surfaces used to test $96.83 \mathrm{~dB}$ re $1 \mathrm{pW}$ lateral quadrupole located at $s_{1}$ and at the sensitivity of the CAA predictions on the FW-H sur$s_{2}$ and radiating at $f=5.67 \mathrm{kHz} . \lambda=0.06 \mathrm{~m}, \ell=1.17 \lambda$, face position.

$a=2.34 \lambda$.

Figure 5. Directivity pattern from a lateral quadrupole and FW-H integration surfaces.

\section{Sensitivity to the FW-H surface dimension}

In this section, the effect of a change in the dimensions of the FW-H surface is analysed, aiming to demonstrate the independence of the results from the integration surface choice. If the surface encloses all the sources of sound and it is placed far enough from the acoustically active region, the solution has to be independent from its shape and position. This property is tested by reviewing the numerical modelling of the sound radiated from the lateral quadrupole source $s_{2}$ presented in section B using FW-H surfaces of increasing extent, as sketched in figure 5(b). The innermost surface is surface $E 1$ sketched in figure 1(b), which was used for the numerical prediction of the sound radiated by a monopole source in section A and by a dipole source in section $\mathrm{B}$. This surface has dimensions $\ell=\lambda$ and $a_{1}=2 \lambda$ and the distance from $s_{2}$ to the surface corner closest to it is $d=0.14 \lambda$. The surface of intermediate extent is the one used for the numerical prediction of the sound propagated from a longitudinal quadrupole and from a lateral quadrupole in section B. This surface has dimensions $\ell=1.17 \lambda$ and $a_{2}=2.34 \lambda$. The distance from $s_{2}$ to the surface corner closest to it is $d=0.43 \lambda$. The outermost surface has dimensions $\ell=1.33 \lambda$ and $a_{3}=2.66 \lambda$ and provides the longest distance from $s_{2}$ to its nearest corner of $d=0.72 \lambda$.

Figure 6(a) shows that using the innermost FW-H surface results in the over-estimation of the sound radiation in the peak directivity direction. Differences in sound pressure amplitude between the analytical reference solution (dashed line) and the numerical predictions (red square symbols) persist at most polar angles. Figure 5(a) shows that increasing the FW-H size to $\ell=1.17 \lambda$ and $a=2.34 \lambda$ allows one to recover the analytical solution in the numerical predictions, as shown by the symbols essentially overlapping the lines in figure $5(\mathrm{a})$.

Figure 6(b) shows that further increasing the FW-H surface extent to $\ell=1.33 \lambda$ and $a=2.66 \lambda$ appears not to produce any significant improvement in the agreement between the analytical and the numerical results. Therefore, using the FW-H surface of intermediate extent provides a prediction that can be considered independent from the FW-H surface size.

\section{Effect of source proximity to the FW-H corners}

Section $\mathrm{C}$ showed that increasing the extent of the FW-H surface enabled us to improve the numerical predictions of the directive sound radiation from source $s_{2}$ that is proximal to the surface corner. This section aims to shed some light on this effect. The characteristic length scales governing the accuracy of the numerical quadrature of the FW-H surface integral are the wavelength $\lambda$ of the radiated sound and 


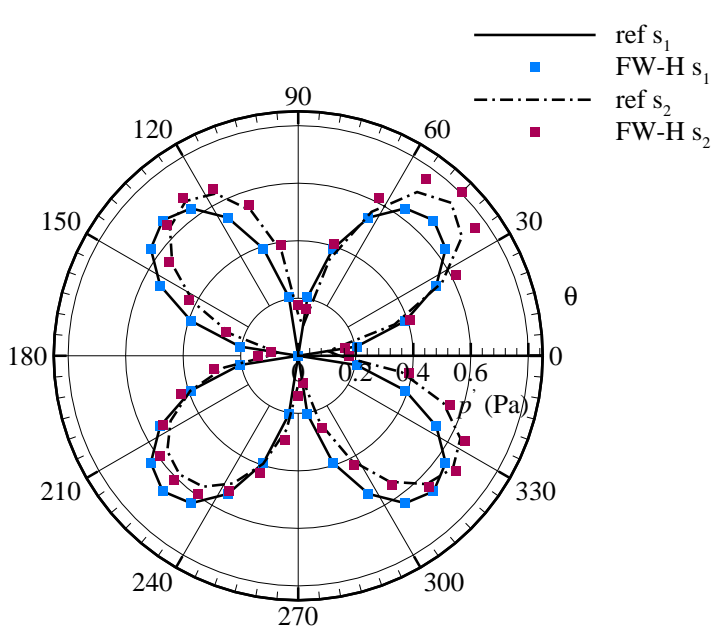

(a) $\lambda=0.06 \mathrm{~m}, \ell=\lambda, a=2 \lambda$.

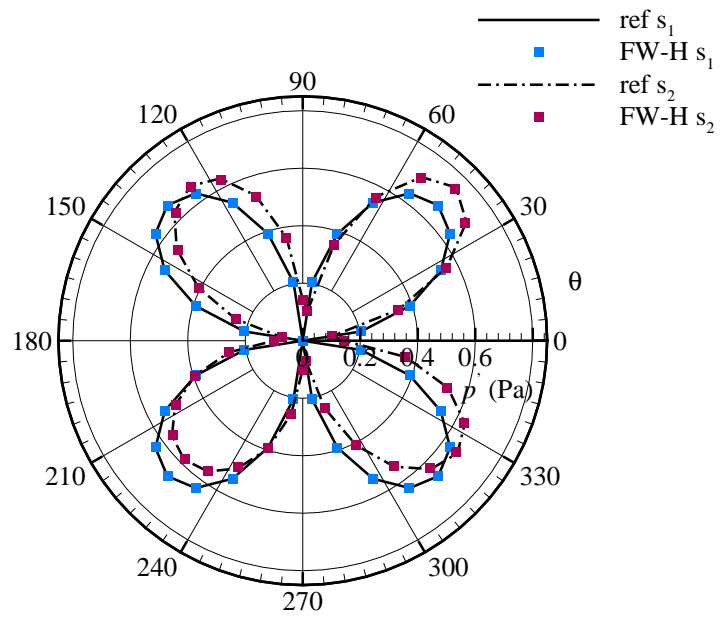

(b) $\lambda=0.06 \mathrm{~m}, \ell=1.33 \lambda, a=2.66 \lambda$.

Figure 6. Effect of the FW-H surface position. Directivity patterns at $10 \lambda$ from the origin from a $96.83 \mathrm{~dB}$ re $1 \mathrm{pW}$ lateral quadrupole located at $s_{1}$ and at $s_{2}$ and radiating at $f=5.67 \mathrm{kHz}$.

the distance between the source and the FW-H surface, which is represented in this study by the distance between a lateral quadrupole centre and its closest corner. In the current implementation of the FW-H postprocessor in Antares, the FW-H surface is discretized uniformly by the spacing $h$. This gives a numerical acoustic wavenumber $k_{a}=h / \lambda$ that expresses how well the acoustic pressure field is resolved in space. The non-dimensional ratio $d / \lambda$ expresses how close the source is to the corner of the FW-H integration surface in terms of acoustic wavelength $\lambda$.

A numerical experiment was conducted by which the position of the lateral quadrupole source $s_{2}$ from section B was systematically varied along the first quadrant diagonal of the FW-H surface of intermediate extent, as sketched in figure 7(a). 40 source positions were considered over the range of $0.845<\lambda / d<17.321$. For each source location, the FW-H surface integration was performed using 50 levels of incrementally finer spatial discretization of the FW-H surface, over the range $0.0147<h / \lambda<0.1944$. At each location, the normalized difference between the reference analytical solution and the numerical prediction of the sound pressure amplitude was computed as

$$
e=\frac{\max \left|p_{a}^{\prime}\left(\theta_{i}\right)-p_{i}^{\prime}\right|_{i=1}^{N_{\theta}}}{\max \left(p_{a}^{\prime}\right)},
$$

where $N_{\theta}=20$ is the number of the observers used to represent numerically the directivity of the lateral quadrupole.

These observers, located at the constant radial distance $r=10 \lambda$, are distributed around the polar arc as shown by the symbols in figure $1(\mathrm{~b}) . \max \left(p_{a}^{\prime}\right)$ normalizes the error by the sound pressure fluctuation amplitude from the lateral quadrupole directivity peak. In the numerical quadrature of continuous functions, the quadrature error often scales exponentially with the level of the discretization. In the current study, the level of the spatial discretization is represented by $k_{a}$. The presence of any such error trend is investigated in figure $7(\mathrm{~b})$ by plotting $\log _{10}(e)$ vs $\log _{10}\left(k_{a}\right)$ and $\log _{10}(\lambda / d)$.

Figure 7(b) shows that, with a spatially well-resolved acoustic sound field, by which $k_{a} \rightarrow 0$, the error iso-contours appear to be regularly spaced from one another, indicating an exponential error decay of order $m=5.64$. As the source $s_{2}$ is located less close to the corner, by which $\lambda / d \rightarrow 0$, then the error iso-contours appear also regularly spaced, indicating an exponential error decay of order $n=1.81$.

The error iso-contour level -2 indicates a $1 \%$ error in the prediction of the acoustic pressure amplitude. This contour line and the lines to the left of it appear to run diagonally parallel to one another. This suggests that a given level of error can be maintained by keeping constant the product $k_{a}^{n}(\lambda / d)^{m}$ so that, for a source located $50 \%$ closer to the $\mathrm{FW}-\mathrm{H}$ surface corner, a spatial refinement of the acoustic domain in the ratio $2^{m / n}$ is required. 


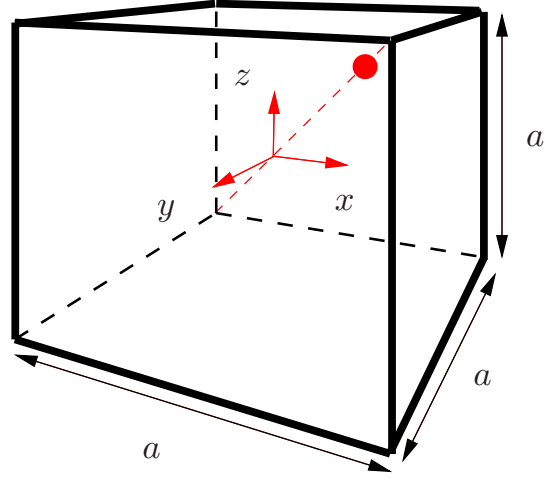

(a) Location range of source $s_{2}$ along the diagonal of the FW-H surface.

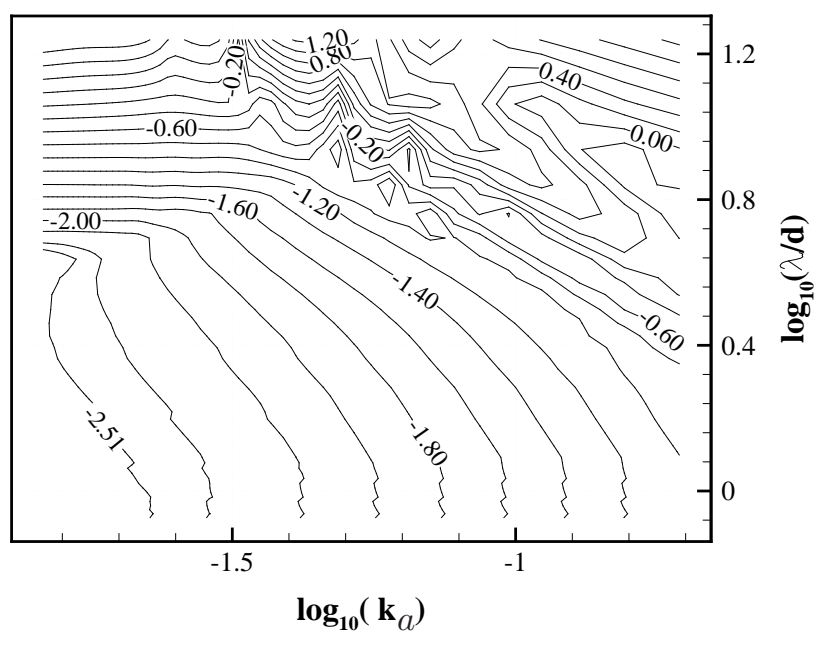

(b) Contours of normalised pressure fluctuation amplitude error plot obtained by placing a lateral quadrupole radiating at $f=5.67 \mathrm{kHz}$ at different locations along the diagonal of the FW-H surface. $N$ number of points per wavelength, $d$ distance source-corner, $\lambda=0.06 \mathrm{~m}, \ell=$ $1.17 \lambda, a=2.34 \lambda$.

Figure 7. Effect of lateral quadrupole source proximity to the FW-H surface corner on the normalised pressure fluctuation amplitude error.

At higher values of the numerical wavenumber $k_{a}$ and of proximity to the $\mathrm{FW}-\mathrm{H}$ surface corner $\lambda / d$, $e$ increases more rapidly and the curvature of the contours in the $\left(k_{a}, \lambda / d\right)$ plane indicates a possible non-linear interaction between these two sources of error.

Figure 7(b) can be used to perform a first estimate of the magnitude of the numerical error that can be expected by a quadrupole source approaching the $\mathrm{FW}-\mathrm{H}$ integration surface corner, for a given level of spatial discretization of the acoustic pressure field. The sensitivity of this error to changes in the source position and in the spatial discretization is also computable.

\section{Conclusions}

A new FW-H acoustic analogy post-processor in the advanced time formulation by Casalino ${ }^{1}$ has been tested on a series of elementary noise sources, showing a good predictive ability in all the tests. Both omni-directional (section A) and directional (section B) sources have been considered. Predictions of the pressure fluctuation amplitude that are substantially independent from the FW-H integration surface have been obtained by an appropriate placement and sizing of the FW-H surface, as shown in section C. Having sharp corners in the FW-H integration surface appears to adversely affect the predictions and this topological feature should be avoided. However, where a sharp corner is unavoidable, the tests have shown that the surface position can be modified to recover the correct noise radiation pattern.

A prediction error analysis from having sharp corners in the FW-H integration surface was performed using a compact quadrupole source located progressively closer to the FW-H surface corner. Error levels of the order of $1 \%$ in directivity are shown to scale exponentially with the spatial resolution of the acoustic field and with the closeness of the source position to the FW-H corner.

This error level may be representable of practical engineering applications, such as the estimation of noise produced aerodynamically by cold subsonic jets, which can be represented by a distribution of convected quadrupoles in the acoustic analogy. This error analysis provides a first estimate for selecting an appropriate FW-H surface layout and for performing a trade-off between surface extent, its placement relative to the closest sound sources, and its spatial discretization.

This validation confirms the ability of the Antares FW-H CAA post-processor to estimate the near-field 
and far-field noise radiation from acoustically compact sources, as provisionally reported by Di Stefano et al. ${ }^{9}$ Further validation will be performed using elementary sources in motion to test the correct prediction of Doppler effects. Multiple sound sources will be then considered, to check that the code correctly sums up the noise contributions emitted at different times and positions.

The new Antares FW-H CAA post-processor is expected to bring advantages to the whole aero-acoustic community. The absence of an upfront license fee aims to support the work of new researchers in Aeroacoustics, who may be pursuing more novel, speculative, and curiosity driven investigations but have yet to secure significant research funds. It is also expected that this communal approach will make the development of the code easier compared to a proprietary software. The Cerfacs team is available to coordinate this development process, making it possible to keep the software up-to-date and to continuously improve its performance and reliability over the years.

\section{Acknowledgements}

The dissemination of these results has received funding from the European Union Seventh Framework Programme FP7/2007-2013 under grant agreement no. 317142. The graphical post-processing of these results was supported by licenses originally purchased with EPSRC support on grant GR/N23745/01.

\section{References}

\footnotetext{
${ }^{1}$ Casalino, D., "An advanced time approach for acoustic analogy predictions," Journal of Sound and Vibration, Vol. 261, No. 4, 2003, pp. 583-612.

${ }^{2}$ Lighthill, M. J., "On sound generated aerodynamically. I. General theory," Proceedings of the Royal Society of London, Vol. 211, No. 1107, 1952, pp. 564-587.

${ }^{3}$ Williams, J. F. and Hawkings, D. L., "Sound generation by turbulence and surfaces in arbitrary motion," Philosophical Transactions of the Royal Society of London, Vol. 264, No. 1151, 1969, pp. 321-342.

${ }^{4}$ Rona, A., Aerodynamic and aeroacoustic estimations of oscillatory supersonic flows, Ph.D. thesis, Department of Aeronautics and Astronautics, University of Southampton, UK, 1997.

${ }^{5}$ Brentner, K. S. and Farassat, F., "Analytical comparison of the acoustic analogy and Kirchhoff formulation for moving surfaces," AIAA Journal, Vol. 36, No. 8, 1998, pp. 1379-1386.

${ }^{6}$ Farassat, F., "Derivation of formulations 1 and 1A of Farassat," Tech. rep., NASA, 2007. 1975.

${ }^{7}$ Farassat, F., "Theory of noise generation from moving bodies with an application to helicopter rotors," Tech. rep., NASA,

${ }^{8}$ Adrien Gomar, T. L. et al., "ANTARES: Python post-processing library, Ver. 1.4.0," http://www.cerfacs.fr/antares/, 2012, CERFACS.

${ }^{9}$ Di Stefano, D., Rona, A., Hall, E., and Puigt, G., "Implementing the Ffowcs Williams and Hawkings acoutic analogy in Antares," The 22 $2^{\text {nd }}$ International Congress on Sound and Vibration, 12-16 July 2015, Florence, Italy, 2015.

${ }^{10}$ Bogey, C. and Bailly, C., "Computation of a high Reynolds number jet and its radiated noise using large eddy simulation based on explicit filtering," Computers \& Fluids, Vol. 35, No. 10, 2006, pp. 1344-1358.

${ }^{11}$ Nelson, P., "Acoustics II," Student notes of M.Sc. course in Sound and Vibration studies, Institute of Sound and Vibration Research, University of Southampton, 1991.
} 